\title{
Docking and Virtual Screening Studies for New Leads of Boar Salivary Lipocalin
}

\author{
Nack-Do Sung, Chang-Sik Park, Hyung-Yeon Park, ${ }^{\dagger}$ and Chan Kyung Kim ${ }^{\dagger}{ }^{*}$ \\ Research Center for Transgenic Cloned Pigs. Division of Applied Biologv \& Chemistrv. Chungnam National Universin, \\ Daejeon 305-764. Korea \\ ${ }^{\dagger}$ Department of Chemistry, Inha University, Incheon $+02-751$. Korea. ${ }^{*}$ E-mail: kckumgomha.ack \\ Received March 21,2008
}

\begin{abstract}
We have performed FlexX docking experiments to predict the best docking poses of 5-androst-16-en-3-ol or 5-androst-16-en-3-one to Boar salivary lipocalin (SAL). Since no steroids were found inside of the binding pocket of the X-ray structure of lGM6. we tried to find docking structures after opening the pocket using the random tweak option implemented in SYBYL. This operation allowed the ligand to enter the pocket. The best poses generated from FlexX were different from the structures reported earlier, which calculated docking poses by manual docking followed by minimization. Analysis of docking poses allowed us to identify phannacophores. From this information virtual screening experiments using UNITY were performed. Among six candidates. 3-(3.7-dimethyloct-6-enylamino)propane-1.2-diol (Leadquest code name: $\mathbf{5 7 5 5}$ ) was chosen for further development. Future work will involve synthesis of some derivatives of $\mathbf{5 7 5 5}$ and biological experiments if any derivatives can control the biostimulation and improve reproductive efficiency in pigs.
\end{abstract}

Key Words : Boar salivary lipocalin. Steroid pheromones. FlexX docking. UNTTY virtual screening

\section{Introduction}

Boar salivary lipocalin (SAL) is a porcine odorant-binding protein (POBP) specifically expressed in the submaxillary glands of the boar. While produced in large amount (hundreds of milligranss) in the gland. it is unable to be detected in the sow. When extracted from the natural sources, this protein is associated with boar's pheromones. Chemical conmunication in the pig has been known for a long time to be mediated by saliva. ${ }^{1}$ In particular, steroid pheromones. such as 5-androst-16-en-3-ol (androstenol, I) and 5-androst16-en-3-one (androstenone. II). present in the saliva of mature males, have been shown to act as pheromones on the female..$^{2}$

In an earlier study by S. Spinelli and coworkers. ${ }^{\hat{}}$ an $\mathrm{X}$-ray structure of SAL was determined $(\mathrm{PDB}$ code $=$ IGM6). Surprisingly. in the $\mathrm{X}$-ray structure a glycerol molecule was found in the binding cavity since the cavity night be too small to accommodate the steroids. Therefore. to obtain the docking poses of I or II to SAL. each ligand was manually introduced into the cavity in two different orientations - $C$ $\mathrm{OH}$ (or $\mathrm{C}=\mathrm{O}$ ) group pointing toward the entrance or the bottom of the cavity. Then each starting structure was minimized to fund an optinum position. As a result of optimization. the volume of the cavity increases due to conformational changes of Val 45 and Ala73. If this is true.
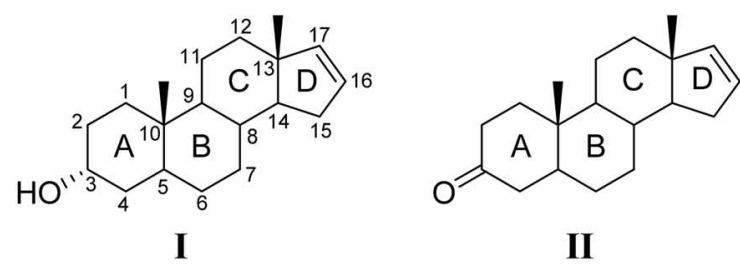

Scheme 1 there still remains a question: how the ligand can enter the binding pocket? To answer this question, we perfomed the docking studies using different approach. namely Flex $\mathrm{X}^{+}$ docking, as our continuing works on computer-aided molecular design. ${ }^{5}$ From the new docking poses, we can find a better way of docking the steroids and of identifying pharmacophores. Using this information, virtual screening study is also carried out to find out new lead compounds. To search of a new porcine pheromonal odorants for biostimulation control system technologies to offer a potentially useful and practical way to improve reproductive efficiency in pigs. this work will be a first step in funding a new active pig pheromonal odorants

\section{Computation}

Since the binding site was not large enough to accommodate one of the steroids. we tried to open it by using the tweak option in SYBYL. Random tweak was developed to predict the conformations of loops in proteins and have been applied successfully in several proteins. ${ }^{6,7}$ By doing this operation, we could open the entrance of the cavity to allow the ligand to enter the binding pocket without problem. In this sense, our approach might be better that earlier one.

FlexX is a fast flexible docking method that uses an incremental construction algorithm to place flexible ligands into a rigid active site and is known to perform well to reproduce X-ray structures. ${ }^{8}$ Standard parameters of the FlexX program as implemented in SYBYL $6.9^{\ni}$ were used during docking. Bond lengths and angles were kept constant as given in the optimized structures. As a preliminary step for docking. structures of I and II were minimized using TRIPOS force field until the root-mean-square (rms) gradient converged to $0.005 \mathrm{kcal} / \mathrm{mol}^{13 i}$ and these ligands were 
docked to the tweaked crystal structure of SAL. FlexX dockings of I and II were performed using the Run-Multiple Ligand option of FlexX. From the best docking poses. important interactions between the ligand and receptor were identified. Based on the analysis of interaction within the binding pocket. we performed virtual screening study using UNITY $^{11}$ module inplemented in SYBYL packages. UNTTY is a search and analysis system for exploring chemical database. Conformationally flexible 3D search rapidly funds molecules that can satisfy queries regardless of the conformation stores in a database. Here, we composed queries based on pharmacophores identified during FlexX docking study. The selected candidates were tested for their toxicity using TOPKAT from Acclerys Inc. ${ }^{12}$

\section{Results and Discussion}

Binding site. In order to perform docking experiment, it was necessary to examine the binding region of the X-ray structure of IGM6. ${ }^{3}$ As mentioned above. the binding pocket formed a closed cavity with a glycerin molecule inside and thus its entrance should be open to accommodate a larger ligand. There were two polar residues at the bottom of the active site (Glu121 and Tyrl23), which could form strong hydrogen bond and/or polar interaction with the ligand The active site was enclosed by several hydrophobic residues such as Val45. Phe58. Ala73, Val85, Phe93. Leu 106. and Leu 108. The active site had volume of $27+\AA^{3}$ and $\log P$ of 15.5 .

To open the binding pocket, we selected the most flexible loop located near the entrance of the protein for flapping. Two loops connecting two $\beta$-sheets were found: loop I was consisted of three residues (Tyr87-Gly89) and loop 2 consisted of 14 residues (Asp30-Val+43). Since loop 1 was too sinall to have sufficient mobility, loop 2 was chosen for this purpose. Mobility of the loop was tested using the tweak option $^{6,7}$ in Sybyl. The conformational change before and after this operation is shown in Figure 1. FlexX docking was

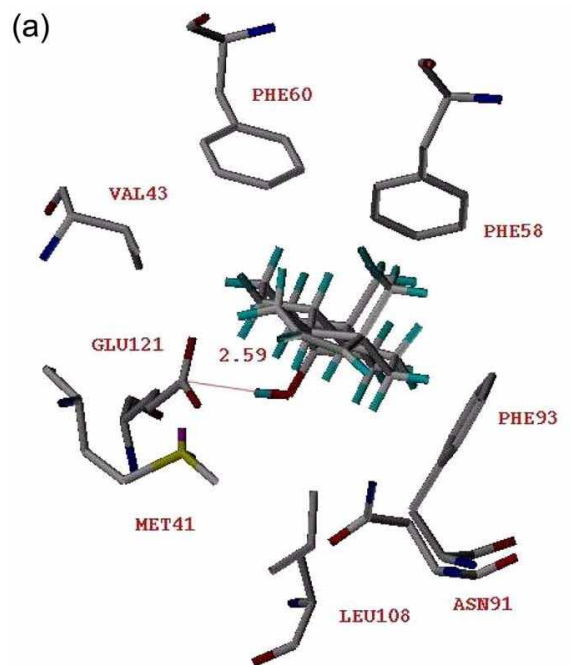

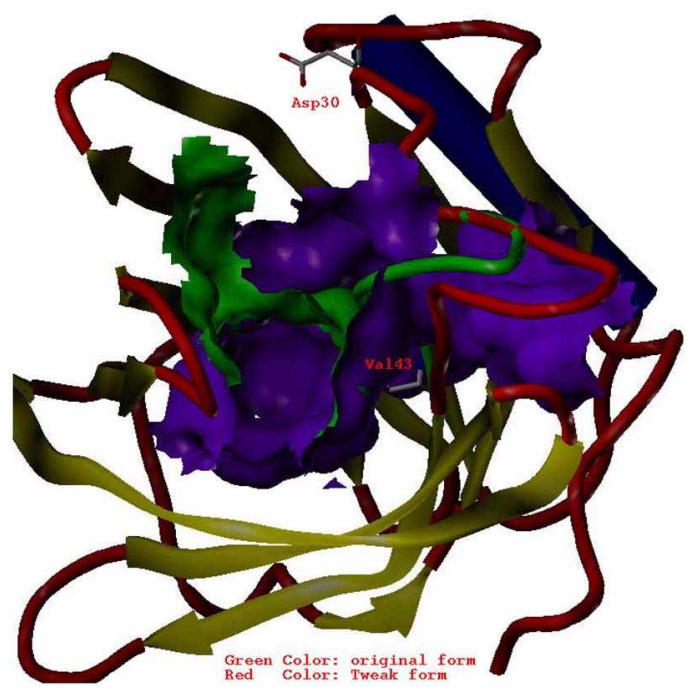

Figure 1. Conformational change of loop2 by random tweak operation.

performed while this loop was open wide.

Docking of androstenol and androstenone. FlexX dockings of I and II were performed after opening the loop 2 at the entrance of the binding region. The best poses are drawn in Figure 2. The two ligands in Figure 2 showed similar orientations in the pocket. The $-\mathrm{OH}$ group of I shown in Figure 2(a) was pointing toward the bottom of the pocket to make a strong hydrogen bond with $-\mathrm{CO}_{2}{ }^{-}$group of Glul21. On the other hand. such an orientation could cause electrostatic repulsion between $-\mathrm{CO}_{2}{ }^{-}$group and $\mathrm{C}=\mathrm{O}$ group of II, C=O group of II moved away from the Glul2I as shown in Figure 2(b). Due to lack of strong hydrogen bond in II. FlexX docking scores of II $(-4.50 \mathrm{kcal} / \mathrm{mol})$ was worse than that of $I(-6.78 \mathrm{kcal} / \mathrm{mol})$. Regardless of the orientations of the ligands, hydrophobic interaction with the surrounding lipophilic residues was a key factor in positioning the ligands in the binding pocket. However, note that the best poses found in this work were different from those

(b)
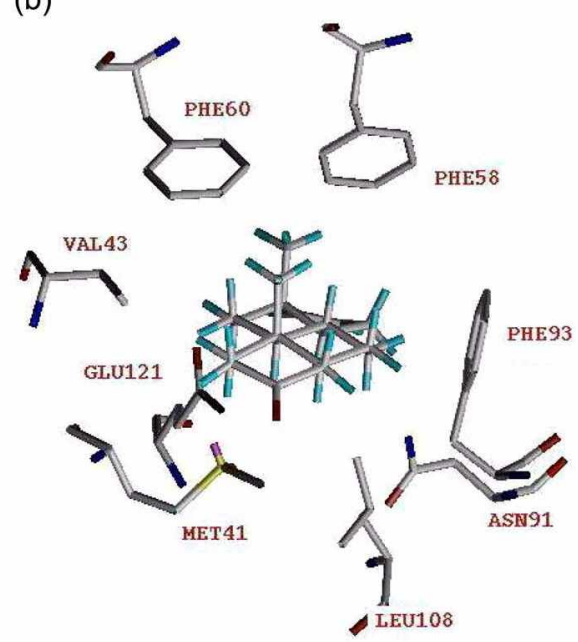

Figure 2. Best docking poses of $\mathbf{l}$ (a) and $\mathbf{l l}$ (b) at the binding pocket of SAL. 


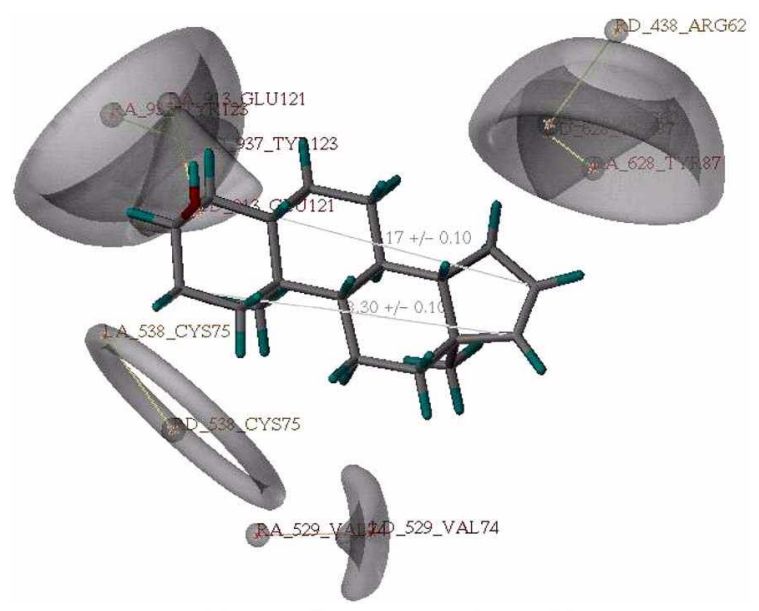

Figure 3. Query option for virtual screening using UNTTY.

reported earlier. ${ }^{3}$ which manually introduced the ligands in the cavity. In an earlier study the polar $-\mathrm{OH}$ group of androstenol was positioned toward the bottom of the cavity but without interaction with the polar residues (Glul21 or Tyrr123) and the polar $\mathrm{C}=\mathrm{O}$ group of androstenone was pointing toward the entrance of the binding pocket. Therefore, we might think that our approach was more rational in that we did not assume any manual operation during docking experiments.

Search for new ligands. From the docking studies. we found that the most important interactions were the hydrogen bonding with Glul21 as well as hydrophobic interaction with residues surrounding the binding pocket. To locate compounds in databases that matched with these pharmaco- phores. virtual screening using UNITY from the docking pose of I was performed. As shown in Figure 3. the following queries were set up: (1) distance constraints $-\mathrm{C}_{2}-\mathrm{C}_{17}$ : $8.30 \pm 0.10 \mathrm{~A}$ and $\mathrm{C}_{4}-\mathrm{C}_{16} \cdot 8.17 \pm 0.10 \mathrm{~A}$ (atom numbering is shown in Scheme 1). (2) receptor donor and corresponding ligand acceptor sites: Arg62. Val74. Cys75, Tyrl23 and Glu121 (3) an additional receptor donor/ligand acceptor site: Tyr87 as hỵdrogen bond acceptor/donor. Three-dimensional molecular candidates were generated by using UNITY from 30.000 entries of the LeadQuest ${ }^{13}$ database from Tripos. 3(3.7-dimethyloct-6-eny lamino)propane-1.2-diol (Leadquest code name: 5755), $\mathrm{N}^{\mathrm{j}}$-allyl- $\mathrm{N}^{2}$-((tetrahydrofuran-2-yl)methyl)oxalamide (LeadQuest code name: 6060). 1-allyl-3-(4(1.1.1.3,3,3-hexafluoro-2-hy droxypropan-2-yl)phenyl)thiourea (LeadQuest code name: 6431). N-[(4-methoxy phenyl)methyl]-3-[[3,5,6,8-tetrahydro-6,6-dimethyl-4-oxo-3-(2propenyl)-4H-pyrano[4'.3'.4.5]thieno[2.3-d]pyrimidin-2-yl] thio]-propanamide (LeadQuest code name: 6663). $\mathrm{N}^{\mathrm{l}}-(4-$ (allyloxỵ)phenyyl)-N²-(2-hỵdroxypropyl)oxalanide (LeadQuest code name: 7111), and 2-(4-methyl-2-oxo-2H-cluromen-7-yloxy)-N-((tetrahyddrofuran-2-yl)methyl)acetanide (LeadQuest code name: $\mathbf{7 1 1 3}$ ) were found as possible candidates and their docking score were evaluated. The docking scores and two typical docking poses are shown in Table 1 and Figure 4, respectively. In Table 1. results of toxiciț prediction using TOPKAT are also summarized. It is interesting to examine the docking poses shown in Figure 4 in detail. The two ligands. 5755 (Figure 4(a)) and 6060 (Figure $4(\mathrm{~b})$ ). fitted well into the binding pocket. However. in the cases of other four compounds. some part of the side chain was located outside of the binding pocket as shown for 6431 in

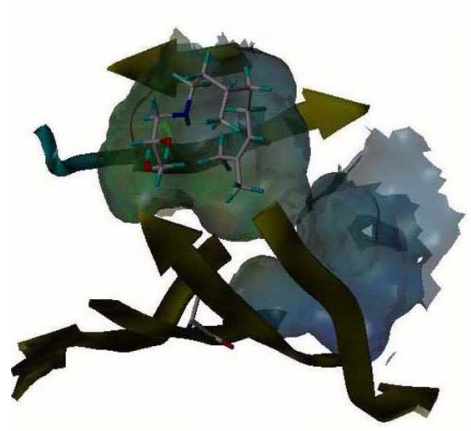

(a)

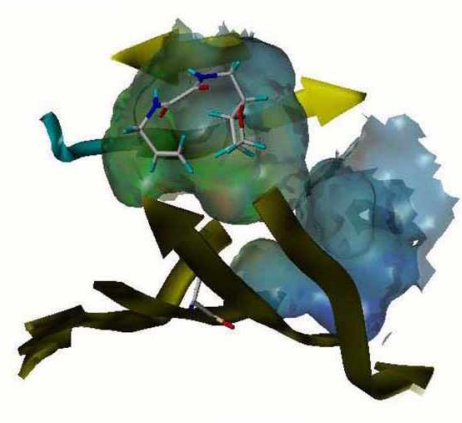

(b)

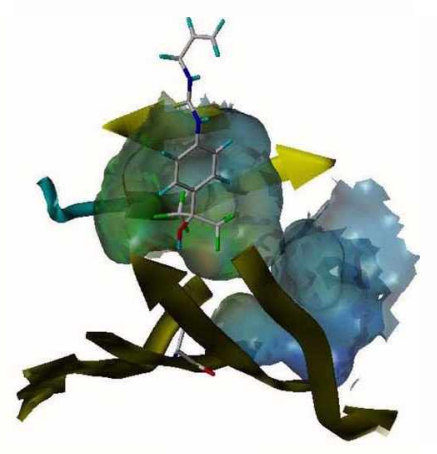

(c)

Figure 4. Three typical docking poses of selected candidates. (a) 5755 (b) 6060 , and (c) 6431

Table 1. Results of docking score and toxicity prediction for six candidates

\begin{tabular}{|c|c|c|c|c|c|c|c|}
\hline Compound No. & Volume ${ }^{b}$ & Docking score $^{\sigma}$ & $\mathrm{FDA}^{c}$ & $\mathrm{AM}^{d}$ & $\mathrm{SI}^{e}$ & $\mathrm{DTP}^{f}$ & $\mathrm{LD}_{50}{ }^{\mathrm{g}}$ \\
\hline 5755 & 227.4 & -39.4 & 0 & 0.00 & 0.06 & 0.00 & $>10$ \\
\hline 6060 & 182.0 & -38.1 & 1 & 0.98 & 0.00 & 000 & 1.8 \\
\hline $6+31$ & 235.4 & -39.9 & 0 & 0,00 & 0.00 & 000 & 201.7 \\
\hline 6663 & 400.5 & -52.7 & 0 & 0,00 & 0.99 & 0.99 & 497.6 \\
\hline 7111 & 2330 & -39.0 & 1 & 0.64 & 0.94 & 000 & 2.8 \\
\hline 7113 & 253.6 & -38.5 & 1 & 0.00 & 0.00 & 0.00 & 2.8 \\
\hline
\end{tabular}

"in kcal:mol. ${ }^{b}$ in $\mathrm{A}^{2}$. ${ }^{c} \mathrm{FDA}$ Rodent carcinogenicity. Non-carcinogen: FDA 0.3 . carcinogen: FDA $>0.7$. Ames mutagenecity. Non-mutagen: AM 0.3 . mutagen: AM $\approx 0.3 .{ }^{e}$ Rabbit skin irritancy. Non-skin irritancy: SI 0.3 , shin irritancy: SI $\% 0.7$. Developmental tovicity potential. No potential for developmental toxicity: DTP 0.3 , potential developmental toxicity: DTP $\%$ ( 7 . ${ }^{\text {Rat }}$ oral LD50 (g:kg) 
Figure $4(c)$. As sumnarized in Table 1, the contputed values of toxicity for $\mathbf{5 7 5 5}$ were determined to be within non-toxic in all dimensions of a model Optinum Prediction Space. Therefore. we selected $\mathbf{5 7 5 5}$ as a possible candidate for further development and experimental studies are in progress in our lab to validate docking/virtual screening results. In future work. we will synthesize some derivatives of $\mathbf{5 7 5 5}$ and perform biological experiments if these compounds can control the biostimulation and improve reproductive efficiency in pigs.

\section{Conclusion}

FlexX docking studies were performed to identify key residues in salivary lipocalin. Before docking. loop 2 was open to make the ligands to enter the binding pocket using tweak option in Sybyl. FlexX docking experiments were performed with Run-Multiple Ligand option. The best docking poses found in the work were different from those found earlier. Using the pharmacophores from docking study. virtual screening using UNITY was performed. Six candidates were identified among 30.000 compounds in the database. One ligand. $\mathbf{5 7 5 5}$, is selected as a lead for further synthesis and examination for biostimulation in pigs.

Acknowledgements. This work was supported by a grant (No. RIl-2002-100-05003) from the excellent research Center of KOSEF.

\section{References}

1. Gower. D. B.: Booth. W. D. Ontog. Olfaction 1986. 255

2. (a) Melrose. D. R.: Reed. H. C.; Patterson, R. L. Br: reteri. J. 1971. 127, 497. (b) Reed, H. C. B.: Melrose. D. R.; Patterson, R. L. S. Br: Veteri. $J$. 1974, 130.61

3. Spinelli. S.: Vincent. R.: Pelosi. P.: Tegoni. M.: Cambillau. C. Eur J. Biochem. 2002. 269. 2449.

4. (a) Rarey: M.: Kramer. B: Lengauer. T.: Klebe. G. J. Mol Biol. 1996. 261,470 . (b) Rarey. M.: Kramer. B.: Lengauer, T. $d$. Comput -Aided Hol. Des. 1997. 11.369.

5. (a) Lee, D. Y.: Hyun. K. H.: Park, H. Y.: Lee, B.-S.; Kim, C. K Bull. Korem Chent. Soc. 2006. 27. 273. (b) Kim. C. K.: Lee. K A.: Zhang. H.: Cho. H.: Lee. B.-S. Bull. Korean Chem. Soc. 2007. 28. 1141. (c) Kim. C.: Kim. J.: Park. H.Y.: McLean. R. T. C.: Kim. C. K.: Jeon. J.; Yi. S.-S.: Kim, Y. G.; Lee, Y.-S: Yoon. J. J. 1ficrobiol Biotedmol 2007. 17, 1598 .

6. Fine. R. M.; Wang. H.: Shenkin. P. S.; Yarmush. D. L.: Levinthal. C. Proteins 1986. 1.342.

7. Shenkin. P. S.: Yarmush. D. L.: Fine. R. M.: Wang. H.: Levinthal. C. Biopolmers 1987. 26.20153.

8. Hoffmann, D; Kramer, B.; Washio, T.: Steinmetzer, T.: Rarey, M: Lengauer. T. J. Med. Chem. 1999, 42. 4422.

9. SYBYL 6.9. TRIPOS Ine.: St. Louis. USA.

10. (a) Powel. M. I. D. Mathentatical Prograntming 1977. 12.241. (b) Clark. M.: Crammer. R. D.: Opdenbosch. N. V. J. Contut. Chent. 1989. 10. 982. (c) Dixon. D. A.: Zeroka. D. J.: Wendoloski. I. J.: Wasserman, Z. R. J. Plys. Chem 1985. 89, 5334.

11. (a) Clark. R. D. J. Chem inf. Compt. Sci. 1997, 37, 1181. (b) Dixon. S. L.: Koehler. R. T. J. 1fed Chem. 1999, 42. 2887.

12. TOPKIT version 6.2: Acclerys Inc.: San Diego. CA, 2001.

13. Lead Ouest Chentical Conpound Libranies: Tripos. Inc:- St.Louis. MO. $2000 \mathrm{r}$ Vol. $1-3$. 